\title{
The 46th annual meeting of the European society for blood and marrow transplantation: nurses group oral session (NO001-NO015)
}

C Springer Nature Limited 2020

29 August - 1 September, 2020 Virtual Meeting

Modified and published with permission from https://www.ebmt.org/annual-meeting

Sponsorship Statement: Publication of this supplement is sponsored by the European Society for Blood and Marrow Transplantation. All content was reviewed and approved by the EBMT Committee, which held full responsibility for the abstract selections.

\section{Nurses Group Oral Session}

NO001.

Family Caregivers Support needs during Allo-hsct - A Longitudinal Study (Preliminary Results)

Annika M Kisch ${ }^{1,2}$, Karin Bergkvist ${ }^{3,4}$, Anette Alvariza ${ }^{5}$, Jeanette Winterling ${ }^{3,6}$

${ }^{1}$ Skåne University Hospital, Lund, Sweden, ${ }^{2}$ Lund University, Lund, Sweden, ${ }^{3}$ Karolinska Institutet, Stockholm, Sweden, ${ }^{4}$ Sophiahemmet University, Stockholm, Sweden, ${ }^{5}$ Ersta Sköndal Bräcke University College, Stockholm, Sweden, ${ }^{6}$ Karolinska University Hospital, Stockholm, Sweden

Background: Family caregivers in allo-HSCT live in a demanding situation, helping the recipient with daily activities and at the same time have to handle their own worries and fear to lose a seriously ill family member. The primary aim of this study was to explore what support needs family caregivers have during an allo-HSCT and how these change over time.

Methods: This was a correlational study with a longitudinal design. A total of 88 family caregivers of patients prior to allo-HSCT at two transplantation centres in Sweden were included from Sept 2017- Dec 2018. Participants filled in a self-administered questionnaire, The Carer Support Needs Assessment Tool (CSNAT) prior to the start of the treatment (baseline) and 3, 6 and 16 weeks after the alloHSCT. The CSNAT has 14 items assessing family caregivers' unmet support needs as providers of care and as people in need of support themselves. It has been developed for assessment of family caregivers' support needs in palliative care, and has been evaluated for validity and reliability in a Swedish palliative context.

Results: The family caregivers were $66 \%$ partners, $18 \%$ children, $10 \%$ parents and $6 \%$ others. Most of them were women $(76 \%)$, lived with the patient $(67 \%)$ and the mean age was 55 years (sd 13.2). The top three support needs among family caregivers reported at baseline were; 'knowing what to expect in the future' (79\%), 'dealing with your feelings and worries' (70\%) and 'understanding your relative's illness' $(65 \%)$. Even though most of the measured support needs in the CSNAT decreased over time, over $50 \%$ of the family caregivers reported that they still had these three support needs at the last follow up. The need of support to know what to expect in the future even increased at 16 weeks.

Conclusions: This is the first time CSNAT has been used in a context of curative treatment. Studies exploring the support needs of HSCT family caregivers are limited in number. Our result is concordant with the few available studies exploring HSCT family caregivers' support needs, indicating that they have unmet information needs, psychological needs and social needs.

Even though family caregivers' support needs decrease over time during an allo-HSCT trajectory over $50 \%$ reported the top three support needs at the last follow-up 16 weeks post HSCT. These results show that family caregivers have a need for more support about the illness and the future, as well as support to handle to live with uncertainty. Using an intervention addressing each family 
caregiver's personal support needs prior to the transplantation might lower family caregivers' strain.

Disclosure: Nothing to declare

\section{NO002.}

Pain and Anxiety Management during Bone Marrow Aspirates in Italian Transplant Programs. A Nationwide Survey on behalf of Gitmo Ng

Gianpaolo Gargiulo $^{1}$, Laura Orlando ${ }^{2}$, Marco Cioce $^{3}$, Valentina De Cecco ${ }^{4}$, Cristina Mentone ${ }^{5}$, Annarita Pesce ${ }^{6}$, Emanuela Samarani $^{7}$, Sonia Soave ${ }^{8}$, Francesca Bonifazi ${ }^{9}$, Fabio Ciceri $^{10}$, Stefano Botti ${ }^{11}$

${ }^{1}$ Federico II University Hospital, Neaples, Italy, ${ }^{2}$ EOC, Bellinzona, Switzerland, ${ }^{3}$ Policlinico Agostino Gemelli, Rome, Italy, ${ }^{4}$ Ospedale Pediatrico Bambino Gesù, Rome, Italy, ${ }^{5}$ Azienda Ospedaliera Mauriziano, Turin, Italy, ${ }^{6}$ Azienda Ospedaliera SS Antonio e Biagio e Cesare Arrigo, Alessandria, Italy, ${ }^{7}$ Spedali Civili, Brescia, Italy, ${ }^{8}$ Policlinico Tor Vergata, Rome, Italy, ${ }^{9} \mathrm{~S}$. Orsola Malpighi University Hospital, Bologna, Italy, ${ }^{10}$ San Raffaele Hospital, Milan, Italy, ${ }^{11}$ Azienda USL-IRCCS, Reggio Emilia, Italy

Background: Bone marrow aspirations (BMAs) are frequent diagnostic procedures in hematology and bone marrow transplant units and patients must often undergo them more than once. The procedure is done manually, introducing a needle through the skin and into the posterior iliac crest or sternum.

Due of its invasiveness, patients frequently experience the BMAs as a frightening procedure and most of them experience pain. Factors influencing pain level including the operators' experience, time and pressure applied as well as insufficient pain relief strategies.

Improved outcome on pain and anxiety management have been achieved introducing and refining sedation procedures. However, sedation is considered mandatory in pediatric settings, while it is poorly considered in adult patients.

Aim of this work was to provide a real-life snapshot on pain management protocols applied during BMAs surveying Italian Transplant Programs.

Methods: A survey questionnaire was developed by GITMO nurses' group and tested for contents, clearness and comprehension in 8 selectioned centres. The survey included two sections regarding BMA practices and nurses' perception (using 1 to 10 likert scale) about applied pain control procedures. The survey was shared using Google Drive to 94 Italian transplant centres; one answer per center was requested. A descriptive analysis was performed on frequencies and central tendency indexes.
Results: From November to December 2018, 59 out of 94 centres $(62.8 \%)$ answered; 13 were paediatric centres, 46 adults. The results showed that 11 TPs $(18,6 \%)$ have not SOPs regarding BMA and 43 centres $(72,9 \%)$ did not have info/educational materials for patients. Before BMAs, info/ educational sessions were provided in all TPs, frequently by the physicians alone $(19 ; 32,2 \%)$. The most used site is the iliac ridge (55 centres; 93,2\%) and that the totality of the centres practices local analgesia prior to the procedure. Before the execution of a BMA, almost none of Italian transplant centres give oral pain therapy $(95,4 \%)$. As per literature, deep sedation is practiced in all paediatric TPs, whereas in adults centres it is largely not used $(29 ; 49,1 \%)$ or only in particular cases $(20 ; 33,9 \%)$, frequently due to patients' request. In addition, when local analgesia is practiced, $22 \%$ of the centres do not respect the time of its action, which leads to huge stress, anxiety, pain and a negative memory-experience to the procedure. Respondants considered BMA as a significative painful (median: 8; SD: 2,7 ) as well as anxiogenic (median: 8; SD: 3,0) experience for patients and they were aware that the adopted techniques were improvable (median: 9; SD: 2,8). On the other hand, in paediatric centers, where BMAs are performed in deep sedation, nurses can focus on the info/educational aspects and the management of anxiety and pain, achieving excellent results for the patients' quality of life.

Conclusions: Management of procedural pain is poorly attentioned in adult settings where often it is considered as inevitable. Starting from the positive experience of pediatric centres, it seems easy to make it possible to reverse the trend for an effective pain and anxiety management. However, high procedural variability and a lack of SOPs were still present in practice.

Disclosure: All authors declare no conflict of interests.

\section{NO003.}

Role of Quicksofa in Predicting Sepsis and Septic Shock in Hematological Patients

Anna Carmagnola ${ }^{1}$, Alessia Arioli, Giulia Righi ${ }^{1}$, Francesca Vergara ${ }^{1}$, Massimo Bernardi ${ }^{1}$, Consuelo Corti $^{1}$, Fabio Giglio ${ }^{1}$, Daniela Clerici ${ }^{1}$, Jacopo Peccatori ${ }^{1}$, Raffaella Greco $^{I}$, Fabio Ciceri ${ }^{1}$, Antonella Biella ${ }^{I}$

\section{${ }^{1}$ IRCCS San Raffaele Milano Scientific Institute, Milan, Italy}

Background: Severe sepsis and multiple organ failure are frequent cause of morbidity and mortality after hematopoietic stem cell transplantation (HSCT) (1). Sepsis is the primary cause of death from infection, especially if not recognized and treated promptly (2). Early diagnosis and 
intervention is fundamental to prevent and manage infections. The quickSOFA score (qSOFA) is a bedside prompt that may identify patients with suspected infection who are at greater risk for a poor outcome (2). It is largely used within intensive care unit. However, its application to hematological patients is not still validated.

Methods: According to Sepsis-3 criteria, sepsis was defined as life-threatening organ dysfunction caused by a dysregulated host response to infection. Septic shock was identified as a clinical construct of sepsis with persisting hypotension requiring vasopressors and a serum lactate level $>2 \mathrm{mmol} / \mathrm{L}(18 \mathrm{mg} / \mathrm{dL})$ despite adequate volume resuscitation. qSOFA and SOFA were defined according to sepsis-3 criteria; qSOFA was calculated according to three criteria based on blood pressure, respiratory rate and mentation state (2).

From October 2018 to September 2019, we conducted a prospective analysis to address qSOFA role in all consecutive adult patients admitted in the hematology and bone marrow transplantation unit. Aim of the study was to determine the utility of qSOFA in the prediction of sepsis and septic shock among hematological patient.

Results: Overall, 202 patients were screened for qSOFA at fever onset; median age was 53 years (range 18-76), 65 females and 137 males. Patients were affected by myeloid malignancies $(n=118)$, lymphoid malignancies $(n=67)$, myeloproliferative disorders $(n=3)$, non-malignant diseases $(n=14)$. Patients received the following treatments: HSCT from MUD $(n=59)$, haploidentical HSCT $(n=43)$, CBU $(n=12)$, autologous HSCT $(n=31)$, chemotherapy $(n=48)$, supportive care $(n=19)$. qSOFA was calculated by nurses at first fever episode (temperature $\geq 38^{\circ} \mathrm{C}$ ), assigning one point for low blood pressure (SBP $\leq 100$ $\mathrm{mmHg}$ ), high respiratory rate ( $\geq 22$ breaths per min), or altered mentation (Glasgow coma scale $<15$ ). If qSOFA was $\geq 2$, the physician calculated the correspondent SOFA score. Another qSOFA was calculated at each criteria variation or in case of new fever episode. We documented a qSOFA at least of 2 points in 35 patients, accounting for 38 episodes considering that 3 patients experienced 2 cases of high qSOFA. The prevalence observed was similar in female $(13,8 \%)$ and male $(21,2 \%)$ patients. Among patients with high qSOFA, severe febrile neutropenia occurred in 23 cases $(60,5 \%)$, and septic shock was observed in 8 cases. Of the 38 cases with high qSOFA, 10 episodes were associated with SOFA inferior to $2(26,3 \%)$ and 28 episodes were associated with SOFA at least of $2(73,7 \%)$.

Conclusions: Hematological patients are very complex, due to different alterations related to underlying disease, treatments and general conditions, able to determine transient and nonspecific alterations in qSOFA criteria. In this analysis, all patient had alterations in qSOFA at the onset of sepsis or septic shock, potentially allowing a quick set of the appropriate monitoring strategies and early antibiotic therapy of the infection. Larger studies are necessary to confirm these preliminary data.

Disclosure: Nothing to declare

\section{NO004.}

A Pathway for Recovery: $D+100$ Autologous Haematopoietic Stem Cell Transplant (Ahsct) follow up Pilot for Lymphoma

\section{Helen Gillespie ${ }^{I}$, Victoria Potter ${ }^{I}$, Shireen Kassam ${ }^{I}$, Andrea Kuhnl', Emma Kane ${ }^{I}$, Michelle Kenyon ${ }^{I}$ \\ ${ }^{1}$ Kings College Hospital NHS Foundation Trust, London, United Kingdom}

Background: Autologous Haematopoietic Stem Cell Transplants (AHSCT) are effective treatment for lymphoma patients at high-risk of disease relapse. JACIE guidelines (FACT-JACIE International Standards Seventh Edition, 2018) recommend clinical policies exist for discharge preparation and structured follow-up. An internal service review recognised AHSCT follow-up care as an improvement area. From December 2018, we piloted an AHSCTspecific follow-up pathway encompassing D0 to D100. The BMT CNS tracked post-discharge blood results and made weekly patient contact (face-to-face/ via telephone) to assess symptoms. At D100, end of pathway holistic needs assessment (HNA) was offered to identify and address unmet needs, support additional signposting and enhance handover information for ongoing follow-up by disease specific teams. A retrospective audit assessed experience of 20 consecutive patients to determine safety, feasibility and SOP adherence before wider implementation.

Methods: December 2018 - June 2019, data were collected on 7 information points outlined in the SOP (Table 1). Findings were collated to determine pathway feasibility, common issues and areas for further development.

Results: 20 consecutive patients commenced the pathway; 2 were withdrawn (1 transferred, 1 incarcerated).

An even gender split $(11 \mathrm{~m}, 9 \mathrm{f})$ was represented, average age 54y (range 33-73y) and most common of 8 lymphoma subtypes transplanted was DLBCL (30\%).

All patients had engraftment accurately tracked and recorded.

Of 18 evaluable patients, planned weekly call completion was $76 \%$. Reasons for non-completed calls include unanswered phones, and pathway cover shortage for BMT CNS annual leave.

Weekly call outcomes included: medication management, other medical speciality and allied health care professional 
referrals, medical review for persistent symptoms or acute deterioration.

Timely line removals were achieved in all eligible patients $(95 \%)$. The remaining patient had poor venous access and required ongoing infusions.

Reasons for disease evaluation non-completion by D100 (4 patients) include non-attendance (2) and appointment unavailability (2).

Patients completing blood tests are weekly 65\%; and fortnightly $30 \%$. Non-completion factors include test ordering issues and Consultant-led cessation

15/18 completed HNA, reporting 96 total concerns (mean 6) with physical concerns fatigue, appetite and taste dominating; and money/ finance being third most reported.

Conclusions: The pathway was broadly adhered to, safe and feasible to deliver.

The pilot highlighted implementation issues that have been addressed; inclusion of follow-up care in BMT CNS cross-covering duties; guidance on Consultant-led blood test cessation at 6-week engraftment review incorporated in SOP revision.

Importantly, routine weekly calls enabled admission prevention due to timely advice, review, symptom management and referrals that may not otherwise have been identified.

HNA provided opportunity for patient reflection, identifying needs, and emphasizing common concerns such as fatigue, anorexia and financial difficulties; areas that will be addressed in treatment preparation, discharge planning and SOP revisions.

Disclosure: Nothing to declare

\section{NO005.}

\section{Prevalence, Predictors and Outcomes of Clabsi Among Hsct Patients}

Jedidah L.M. Lieow ${ }^{1}$, Pin Yan Tan', Yee Mei Lee ${ }^{1}$, Mary Jeanette Jacinto Ignacio ${ }^{2}$

${ }^{1}$ National University Cancer Institute, Singapore, Singapore, ${ }^{2}$ National University of Singapore (NUS), Singapore, Singapore

Background: Central line-associated bloodstream infection (CLABSI) is a significant risk factor of mortality after hemopoietic stem cell transplant (HSCT). There is scarcity of research that identifies the incidence rate and predictors of CLABSI specifically in adult HSCT population.

AIM of the study was to

1) Describe demographic and clinical characteristics of HSCT patients with CLABSI

2) Compare and identify predictors for CLABSI
3) Evaluate patient outcomes as a result of CLABSI

Methods: An observational retrospective chart review single-center study was conducted on HSCT patients from 1st July 2016 to 31 st October 2017 using total population sampling. It is approved by institutional review board with a waiver of consent obtained. Data analysis was performed using univariate analysis and logistic regression on Statistical Package for Social Sciences Version 25.

Results: Total 103 autologous and allogeneic HSCT patients, with any types of CVC were included in the studies. A total of 65 episodes of CLABSI were recorded, resulting CLABSI rate of 5.62 per thousand central line days.

Univariate analysis indicated statistical significance in cancer diagnosis $(p<0.001)$, conditioning regime $(p=$ $0.002)$, graft type ( $p=0.043)$, donor type $(p=0.004)$, central venous catheter (CVC) type $(p=0.002)$, CVC site $(p=$ $0.042)$, history of CLABSI $(p<0.001)$, presence of line occlusions $(p<0.001)$, neutropenia during CLABSI $(p<0.001)$, blood products transfusion within 7 days before onset of CLABSI $(p<0.001)$ and duration of CVC $(p=0.03)$. However, logistic regression only concluded CVC type, history of CLABSI, presence of line occlusions and cancer diagnosis as predictors of CLABSI. The etiology of CLABSI was revealed to be mainly gram-negative bacteria (GNB) and multidrug resistant organisms (MDRO).

$45.2 \%$ of the CLABSI cases fulfilled the definition for mucosal barrier Injury laboratory-confirmed bloodstream infection (MBI-LCBI) and these patients had clinical symptoms before ICU transfer, resulting CVC removal.

\section{Conclusions: DISCUSSION}

The three most prevalent findings in this study includes

1. History of CLABSI and presence of line occlusions as predictors of CLABSI

2. Association of blood transfusion within 7 days before onset of CLABSI

3. Etiology of CLABSI are mainly GNB and MDRO

Antimicrobial prophylaxis regime should be relooked, especially in an era of antibiotics resistance. The association between line occlusions and CLABSI in the study suggests prevalent re-infections. As this is a relatively new finding, more studies are required to validate this finding.

The data collection for this study was completed just before the release of revised CLABSI guideline by Centre for Disease Control and Prevention (CDC). Our institution has made a few evidence-based practice changes during the study (including robust skills training for medical professionals) and results post interventions are underway.

Conclusion: Interesting findings of line occlusions and history of CLABSI, as predictors of CLABSI, illuminated areas for further studies to validate these uncommon 
findings. The CLABSI pathogens identified have also highlighted clinical practices that can be improved.

Clinical Trial Registry: NHG DSRB Ref: 2018/00835

https://www.research.nhg.com.sg

Disclosure: Nothing to declare

\section{NO006.}

\section{Creation of the Labmt Nurses Group}

Mariela Blanco $^{1}$, Eugenia Trigoso ${ }^{2}$, Julia Ruiz ${ }^{3}$, Ana Grace Barrantes ${ }^{4}$, Sara Saez ${ }^{5}$, Paola Viveros ${ }^{6}$, Lidiane Soars $^{7}$, Lucia Vazquez ${ }^{8}$, Tania Waisbeck ${ }^{7}$, Cristina Vogel $^{7}$, Gregorio Jaimovich ${ }^{9}$, Michelle Kenyon ${ }^{10}$, Sebastian Galeano ${ }^{11}$

${ }^{1}$ Hospital Universitario Fundacion Favaloro, CABA, Argentina, ${ }^{2}$ Hospital Universitari $i$ Politècnic La Fe, Valencia, Spain, ${ }^{3}$ Hospital del Niño Jesus, Madrid, Spain, ${ }^{4}$ Hospital, Costa Rica, Costa Rica, ${ }^{5}$ Hospital ClínicCalvo Makena, Santiago de Chile, Chile, ${ }^{6}$ Hospital Calvo Makena, Santiago de Chile, Chile, ${ }^{7}$ Hospital Albert Einstein, Sao Paulo, Brazil, ${ }^{8}$ Hospital, Asuncion, Paraguay, ${ }^{9}$ CEHT, Buenos Aires, Argentina, ${ }^{10}$ King's College Hospital NHS, London, United Kingdom, ${ }^{11}$ Hospital, Montevideo, Uruguay

Background: A new collaborative Nurses group has been initiated from the Latin American Blood and Marrow Transplant group (LABMT) in August 2019. The key objectives of our Nurses group are to promote new developments, education, collaboration and participation of nurses working in the field of Hematopoietic Stem Cell Transplantation (HSCT) in Latin America, as well as develop collaborative opportunities with international HSCT nursing bodies.

Methods: The formation of the group began in July 2017, and at the beginning this group was focused on nurse training for quality, based on FACT-JACIE standards and continued evolving. [J1] Two coordinators and secretaries were appointed for Spanish and Portuguese-speaking countries. A regional strategy was created, identifying a representative for each of the participating countries. This process was supported by members of the European Society for Blood and Marrow Transplant Nurses Group (EBMT-NG).

Results: Initially, representatives from 14 Latin American countries were convened to form the LABMT-NG. The number of members from these countries has since increased to 254 to date. Webex (conference call application) was [KM1] used for online meetings and webinars. [J2] A number of events have been delivered to date. In 2018, 4 joint LABMT-EBMT-NG webinars were carried out. In August 2019, LABMT Nurses Group Inaugural
Meeting was held during the 3rd Meeting of the LABMT / XX1ll SBTMO in Brazil, where 300 local nurses and representatives from most countries participated. In September 2019, the first LABMT Nurse Study day was held in Paraguay with the 6th WBMT / WHO Workshop, with 65 attendees from the region.

Conclusions: The achievements of the LABMT-NG have inspired them to formalize their status. They have finalised the terms of reference. Future priorities have been identified: creating tools to collaborate in nurse education in HSCT in Latin America. Accompanying this priority is a project to offer 10 monthly educational webinars in 2020 , actively participate in the global meetings: EBMT 2020 in Madrid, Spain, SBTMO 2020 in Sao Paulo/Brazil and LABMT 2021 in Montevideo/Uruguay.LABMT-NG first steps have resulted in valuable interaction between the coordinators, local representatives and representatives of the EBMT as well as other LABMT Committees. There is common ground in the need for continuous education. Despite the distances and languages, we have demonstrated effective collaboration and developed a clear action plan for the future of the LABMT Nurses Group.

Disclosure: Nothing to declare

\section{NO007.}

The effect of Motivational Interview Performed after Stem Cell Transplantation on Sexual Experience, Sexual Self-awareness and Sexual Self-efficacy

\section{Gunay Dikbiyik ${ }^{1}$, Esra Koroglu Camdeviren ${ }^{2}$}

${ }^{1}$ Medipol University Hospital, Bağcılar, Turkey, ${ }^{2}$ Medipol Üniversitesi, Istanbul, Turkey

Background: Hematopoietic stem cell transplantation (HSCT) is widely used in the treatment of some benign and malignant disorders. The aim of our study was to investigate the effect of motivational interview after stem cell transplantation on sexual experience, sexual self-consciousness and sexual self-efficacy.

Methods: The study was designed as a prospective randomized controlled trial and conducted on patients who underwent HSCT at a private instute, located in Istanbul. Accrual of the participants started at May 2017 and ended on February 2019. A total of 62 patients, who gave a written informed consent, were randomized into two groups. First group $(n=31)$ included patients who were intervened by the researcher and the other $(n=31)$ included un-intervened controlled subjects. Data regarding the clinic and demographic features of the participants were collected using the BMT unit registry database. Sexuality Characteristics Data Collection Form, Arizona Sexual Experiences Scale (ACSS), 
Sexual Self-Consciousness Scale (CISS) and Sexual SelfEfficacy Scale were used to collect the data among the sexual outcomes. The researcher provided motivational interviewbased counseling services to the intervention group patients three times. The first was on the day of discharge, the second and third interviews were on the first week after discharge and the first month after discharge, consequently. No intervention other than routine discharge training was applied to the control group patients. At the end of the trial, motivational interview was applied to the control group after the last test due to the ethical aspect of the research.

Results: Of the 62 patients who participated in the study, 29 were under 40 years old, 33 were 40 years and over. 27 were male, 35 were female and 29 were underwent allogeneic HSCT and the remaning went on autologous HSCT. $58.1 \%$ of patients in the intervention group and 51.6 $\%$ of control group patients underwent an allogeneic HSCT. We showed that motivational interviewing had no significant effect on sexual experience $(\mathrm{p}=$.) and it did not create a significant difference on sexual self- awareness $(\mathrm{p}=)$ and sexual self-efficacy when compared with observation only.

Conclusions: Despite the fact that we were not able to document a significant beneficial effect of sexual motivational interview, we believe that sexual counseling should be integrated into the training of nurses who specifically deal with the care of patients who undergoes HSCT. Forms to evaluate the sexual problems of the patients should also be a part of the nursing forms and they should be organized and collected institutionally in a regular basis, as they are regarded as an important parameter of the quality of life. Also training courses among this particular subject should be planned to educate the caregivers outside the hospital.

Disclosure: Nothing to disclose.

\section{NO008.}

\section{Symptoms and Quality of Life of Children and Adolescents undergoing Hematopoietic Stem Cell Transplantation or Chimeric Antigen Receptor T Cell Therapy}

\section{Jessica Ward ${ }^{1}$, Paula Murray ${ }^{1}$, Kimberly Powers ${ }^{2}$, Jilayne Smith $^{3}$, Melody Hellsten ${ }^{4}$}

${ }^{1}$ Childrens' Hospital Los Angeles, Los Angeles, CA, United States, ${ }^{2}$ Ann and Robert H. Lurie Children's Hospital of Chicago, Chicago, IL, United States, ${ }^{3}$ Children's Hospital of Colorado, Aurora, CO, United States, ${ }^{4}$ Texas Children's Hospital, Houston, TX, United States

Background: Hematopoietic stem cell transplantation (HSCT) and chimeric antigen receptor (CAR) T-cell therapy are potentially curative therapies for children with life- threatening conditions, but can result in a high symptom burden and poor quality of life (QoL). Parents provide extensive care for their children and can experience distress, yet the association between parent and child outcomes has not been sufficiently investigated. The purpose of this study is to describe the trajectory of symptom burden and QoL among children undergoing HSCT or CAR T-cell therapy, and to determine the impact of parent distress.

Methods: This multisite study employed a longitudinal, repeated measures design. English or Spanish-speaking children ages 2-18 years, with any underlying diagnosis, and planned HSCT or CAR T-cell therapy were eligible. Children completed the Memorial Symptom Assessment Scale (MSAS) and the PedsQL Cancer Module at 4 timepoints: pre-HSCT or CAR T-cell infusion, and day $+30,+60,+90$ post-infusion. Parents completed MSAS and PedsQL proxy for younger children. Parent distress was measured using the Beck Anxiety and Depression Inventories, the Perceived Stress Scale and the PROMIS Sleep and Fatigue Short Forms at corresponding times. Descriptive statistics, correlation analysis and multilevel modeling were used to explore the trajectory of child symptom burden, QoL, and relationships between parent distress (a single factor consisting of anxiety, depression, stress, sleep and fatigue) and child outcomes. Multilevel regression analyses were done to determine predictors of QoL and symptom burden, respectively.

Results: Children and adolescents $(n=140)$ were enrolled across 4 participating institutions in the United States. Child mean age was 8.3 years $(\mathrm{SD}=4.9), 55.4 \%$ were male, primarily with a diagnosis of malignancy (66.9\%). Most parents were mothers (79.1\%), mean age of 38.9 years $(\mathrm{SD}=8.1)$. Child QoL was lowest and symptom burden was highest pre-cell infusion for children receiving HSCT or CAR-T cell therapy. Child symptom burden and QoL improved from pre-cell infusion through day +90 . There was a negative correlation seen between symptom burden and QoL. Children with high QoL had low symptom burden, although a wide range of symptom burden was reported for children with low QoL (Figure 1). Multilevel regression analysis revealed that time post-cell infusion was the only significant predictor of symptom burden and QoL with no significant effect of child age, gender, diagnosis and therapy (autologous HSCT, allogeneic HSCT, or CAR-T). Parent distress scores were consistently higher than reference values for each measure. When parent distress was higher at baseline, child QoL was lower. As parent distress increased over time, child QoL decreased. Parent distress was not associated with child symptom burden.

Conclusions: Children can experience high symptom burden and low QoL, particularly prior to cell infusion. Findings also suggest that parents experience heightened distress throughout their child's HSCT or CAR T cell therapy and their distress is associated with child QoL, but 
not symptoms. Routine, comprehensive symptom assessment with multimodal interventions are needed to improve the symptom experience for children undergoing HSCT or CAR T-cell therapy. Increased psychoeducational support tailored to address parental distress is necessary and has potential to positively impact child QoL.

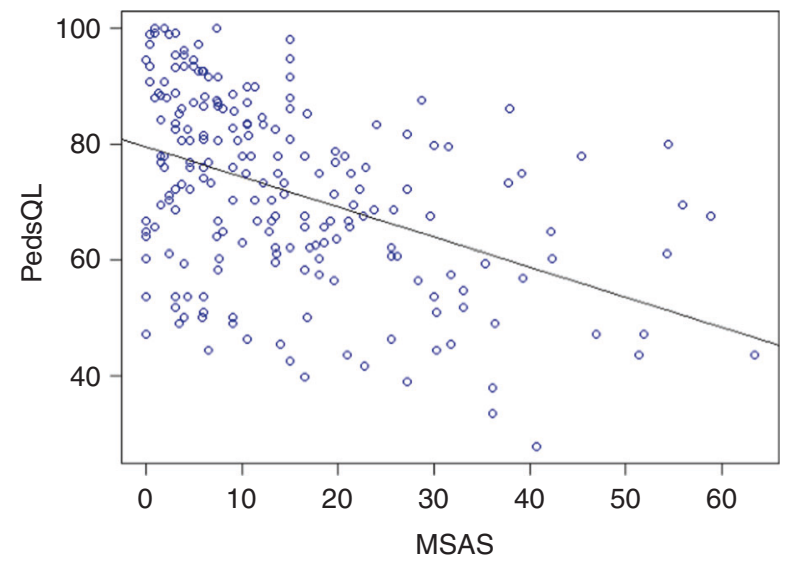

[Figure 1: Symptoms and Quality of Life]

Disclosure: Nothing to declare

NO009.

Social Support Moderates the effects of Health Stressors on Health Care Adherence and Psychological Distress in Long-term Hematopoietic Stem Cell Transplantation (Hsct) Survivors

\section{Kristina Nørskov ${ }^{1,2}$, Jean C. Yi ${ }^{3}$, Marie-Laure Crouch ${ }^{3}$, Karen L. Syrjala ${ }^{3,4}$}

${ }^{1}$ Rigshospitalet, Copenhagen, Denmark, ${ }^{2}$ University of Copenhagen, Copenhagen, Denmark, ${ }^{3}$ Fred Hutchinson Cancer Research Center, Seattle, WA, United States, ${ }^{4}$ University of Washington, Seattle, WA, United States

Background: Advances in the treatment with Hematopoietic Stem Cell Transplantation (HSCT) have improved survival rates with an increasing number of long-term survivors. However, the treatment with HSCT still remains one of the most toxic treatments for hematological malignancies with a potential severe effect on physical and psychosocial functioning which further can impact their quality of life. Poor social support has been linked with physical morbidity and mortality as well as psychological distress. This study tested a theory-driven hypothesis that social support buffers adverse effects of health stressors on psychological distress among long-term HSCT survivors, and that buffering effects of social support would be associated with selfefficacy and adherence to recommended health care.
Methods: The design is a cross-sectional secondary analysis of a randomized controlled trial using baseline, pre-randomization data and prospective medical record data. All eligible adult survivors at a large transplant center were approached if they were 3-18 years after HSCT and treated for hematologic malignancy without relapse or second cancer in the previous 2 years. Data included medical records and patient-reported outcomes (PRO) including Cancer and Treatment Distress (Distress) and Health Care Adherence (Adherence) to HSCT-specific survivor recommendations. Validated, reliable, PRO included health stressors: Comorbidity Index (Comorbidities) and active cGVHD. Social support moderators included: ENRICHD Social Support Instrument (Support) and Social Activity Log (SAL). The cognitive process moderator was Health SelfEfficacy. Established cut points, where available, defined 'impaired' cases. We tested two hypothesized models for Adherence and Distress with blocked hierarchical linear regressions: block 1 demographic/clinical covariates, block 2 health stressors, block 3 hypothesized social moderators, block 4 hypothesized cognitive process moderator.

Results: The 781 participants were $44 \%$ female, with mean age of 52 (range 18-79 years); 64\% were $<10$ years posttransplant; 75\% received allogeneic HSCT. Comorbidities $>3$ were present in 38\%; $8 \%$ had active moderate-severe cGVHD; 30\% reported low Support; 30\% reported elevated Distress; and $49 \%$ reported low Adherence $(\leq 80 \%)$. Regression models found that high support and SAL moderated the effect of health stressors and that Self-Efficacy added significantly to the models for both adherence (full model $\mathrm{R}^{2}=0.25, p=<0.001$ ) and distress (full model $\mathrm{R}^{2}=0.35, p=<0.001$ ).

Conclusions: The two tested models confirm that the health stressors of comorbidities and cGVHD are moderated by better social support and self-efficacy and are associated with lower psychological distress and greater health care adherence. These results provide important theoretical and clinical evidence from which to target interventions to enhance the care of long-term survivors of HSCT.

Clinical Trial Registry: clinicaltrials.gov NCT00799461

Disclosure: Nothing to declare

\section{NO010.}

\section{Study on Cognitive Function and Related Factors after Hematopoietic Stem Cell Transplantation}

\section{Yun Fang ${ }^{1}$, Xiaorong Zhang ${ }^{1}$, Minjie Liu ${ }^{1}$, Weiwei Zhang ${ }^{1}$}

${ }^{1}$ Tongji Medical College of Huazhong University of Science and Technology, Wuhan, China

Background: Few studies have examined the effects of 
hematopoietic stem cell transplantation (HSCT) on the cognitive function of patients with hematologic malignancies. The aim of the study was to assess cognitive performance and analyze the possible influencing factors. The correlation between fatigue, anxiety, depression and cognitive function in patients with HSCT were determined.

Methods: A consecutive sample of 160 patients was assessed after HSCT. The Chinese version of the Functional Assessment after Cancer Cognition (FACT-Cog), Functional Assessment of Chronic Illness Therapy-Fatigue (FACIT-F), Hospital Anxiety and Depression Scale (HADS) were administered. Statistical method of descriptive statistical analysis, independent sample t-test, one-way analyses of variance (ANOVAs), nonparametric analysis, spearmen correlation analysis, and multiple linear regression analysis were used to determine factors associated with fatigue, anxiety, depression and cognitive function.

Results: In this study, 170 questionnaires were sent out and 160 questionnaires were recovered, with 152 valid questionnaires $(89 \%)$ were collected. The total score of cognitive function were $(120.57 \pm 21.319)$, and dimension score of perceived cognitive impairment, other people's evaluation, perceived cognitive ability and impact on quality of life were $(65.47 \pm 11.961),(14.43 \pm 2.278)$, $(26.78 \pm 7.062)$ and $(13.95 \pm 3.176)$, respectively. The score of fatigue, anxiety, and depression were (38.19 \pm $8.163),(5.64 \pm 2.695)$, and $(3.45 \pm 3.168)$, respectively. The correlation analysis showed that fatigue was most related to cognitive function $(r=0.264, p<0.01)$. Anxiety and depression were related to cognitive function $(\mathrm{r}=-0.090, p<0.05 ; r=-0.308, p<0.05)$. The multiple linear regression analysis showed that Gender, age, occupation, fatigue and other independent variables could explain $33.5 \%$ of the total variation of cognitive function $(\mathrm{F}=6.173, p<0.001)$. Gender, age and fatigue can be used as predictors of cognitive function. Gender can explain $3.5 \%$ of total variation of cognitive function $(\mathrm{F}=5.267, p<0.05)$, age can explain $10.6 \%$ of total variation of cognitive function $(\mathrm{F}=11.976, P<0.05)$, and fatigue can explain $25.2 \%$ of the total variation of cognitive function $(\mathrm{F}=31.328, p<0.001)$.

Conclusions: This study showed cognitive impairments in patients with HSCT, and gender, age and fatigue can be considered as predictors for cognitive function, among which fatigue plays an important role in prediction. It is also necessary to know whether it is possible to intervene with changeable factors such as occupational status, sleep status, fatigue, anxiety, depression, and so on, so that we can provide the effect intervention to prevent and improve cognitive dysfunction.

Clinical Trial Registry: No

Disclosure: Nothing to declare
NO011.

Symptom Distress during the First Year after Allogeneic Hematopoietic Stem Cell Transplantation - A Prospective Study

\author{
Linda Victoria Eriksson ${ }^{1,2}$, Katarina Holmberg ${ }^{1,3}$, Karin \\ Bergkvist ${ }^{1,3}$, Carina Lund Hagelin ${ }^{1,4}$, Jeanette \\ Winterling $^{1,2}$
}

${ }^{1}$ Karolinska Institutet, Stockholm, Sweden, ${ }^{2}$ Karolinska University Hospital, Stockholm, Sweden, ${ }^{3}$ Sophiahemmet University, Stockholm, Sweden, ${ }^{4}$ Ersta Sköndal Bräcke University College, Stockholm, Sweden

Background: Patients are affected by various symptoms in the aftermath of an allogeneic hematopoietic stem cell transplantation (allo-HSCT). Previous research focuses mainly on symptom occurrence, although the most frequently occurring symptoms may not be what the patients find the most distressing. By exploring the distress of symptoms, rather than the occurrence, care for this patient group can be improved to include support for the symptoms they find most distressing.

The aim of this study was to describe how patients experience symptom distress during the first year following an allo-HSCT.

Methods: Patients between the ages of 18-65 who underwent an allo-SCT between 2009-2016 due to a haematological malignancy were included $(n=189)$. Questionnaire data were collected at allo-HSCT, four, seven and 13 months after allo-HSCT. Symptom occurrence and distress were measured using the Symptom Frequency, Intensity, and Distress Questionnaire for Stem Cell Transplantation (SFIDSCT) consisting of 38 symptoms in which the most frequent and distressing symptoms were ranked.

Results: Data were analysed using descriptive statistics and preliminary results show that self-reported symptom distress changes during the first year after allo-HSCT. At allo-HSCT, the symptoms causing the most distress were difficulties eating (67\%), discomfort during sexual intercourse $(55 \%)$ and mouth ulcers $(52 \%)$. Four months after treatment, the most distressing symptoms were difficulties breathing (52\%), lack of energy (50\%) and difficulties eating $(50 \%)$. At seven months, sexual potency for men (54\%), poor physical fitness $(50 \%)$ and fragile genital membranes (49\%) were the most distressing symptoms, while at 13 months, the symptoms causing the most distress were discomfort during sexual intercourse (53\%), difficulties eating (51\%) and pain $(50 \%)$. The highest scoring occurrence of symptoms at 13 months after allo-HSCT were tiredness $(86 \%)$, poor physical fitness $(82 \%)$ and lack of energy $(80 \%)$. However, these symptoms were not the 
symptoms causing the most distress as only $38 \%$ scored high distress for tiredness and poor physical fitness, and $41 \%$ scored highly on distress for lack of energy 13 months post treatment. Further analyses are in progress and more data will be presented at the conference.

Conclusions: The symptoms patients experience the most do not necessarily cause the most distress and what was considered as distressing varied between participants. Further, the most distressing symptoms changed over time during the first year following allo-HSCT. Health care professionals, especially nurses, have an important role in helping patients manage their symptoms, and the discrepancy between symptom occurrence and symptom distress must be considered during the rehabilitation phase. By improving the understanding of symptom distress, nurses will be better equipped to support patients anticipate their symptoms and hence, implement early management strategies.

To avoid unnecessary suffering for allo-HSCT patients, health care professionals should focus on what the patients find most distressing in the aftermath of their treatment, rather than the most prevalent symptoms, and address these symptoms during rehabilitation.

Disclosure: There are no conflicts of interest to disclose.

\section{NO012.}

\section{The Effect of Relaxing Breathing Exercises on Pain, Fatigue and Leukocyte Count in Adult Patients Undergoing Hematopoietic Stem Cell Transplantation}

\section{Esra Bayrak ${ }^{1,2}$, Gulbeyaz Can ${ }^{2}$}

\section{${ }^{1}$ Anadolu Medical Center Hospital, Kocaeli, Turkey, ${ }^{2}$ Istanbul University, Istanbul, Turkey}

Background: Patients who underwent hematopoietic stem cell transplantation; have to cope with many symptoms that adversely affect their daily life activities during the period of chemotherapy, stem cell transplantation and subsequent engraftment. The studies related to the chemotherapy-related symptoms of hematological cancer patients showed that fatigue with $97.5 \%$ and the pain with $53.6 \%$ are the most common symptoms experienced by the patients. It was also shown that the white blood cell (WBC) value was the lowest when the symptoms were most severe. This experimental study is aimed to investigate the effects of relaxing breathing exercises on pain, fatigue and leukocyte count in patients undergoing hematopoietic stem cell transplantation.

Methods: The study was conducted between October 2017-August 2018 with 67 volunteer patients who were treated at the Bone Marrow Transplantation Unit of the Private Anadolu Medical Center Hospital. 30 patients were included in the experiment group and 37 patients were included in the control group using the randomization list prepared previously. All patients included in the study were informed about the purpose and application of the study. The experimental group was taught and practiced relaxing breathing exercises with a 20-minute by an instructor for five days a week. Patient Assessment Form, The Visual Analog Scale (VAS), Brief Pain Questionnaire, and Visual Similarity Scale for Fatigue (YIBO) were used as assessment instruments.

In the statistical evaluation of the data, Student's t, Mann Whitney U, Kruskal Wallis, Spearman Rho Correlation Analysis, Pearson's Chi-Square Test, Continuity (Yates) Corrected Chi-Square Test, Fisher's Full-Square Test, and Shapiro Wilks Test were used.

Results: Regarding demographic characteristics of the patients; most of them were male (64.2\%), married (78\%) and have children (78\%), and their education level was primary or lower level $(49.2 \%)$. When fatigue and energy levels of the patients were compared, it was found that the fatigue level of the patients in the control group was higher and the energy level was lower than the patients in the experimental group. Moreover, female patient population experienced more fatigue, while the fatigue level of the female patients in the experimental group was low. With regards to the effect of relaxing breathing exercises on discharge process, the discharge time of the autologous and allogeneic transplantation patients in the experimental and control groups was compared. It was found that the discharge process of the allogeneic transplantation patients in the experimental group was shorter than the patients with allogeneic transplantation in the control group $(p=0.04)$.

Conclusions: To conclude, it was observed that relaxing breathing exercises have positive effects on fatigue management and trough these exercises it is possible to shorten the discharge time of patients with allogeneic transplantation. On the other hand, there is no statically meaningful evidence that the relaxing breathing exercises have affect on the pain and immune system the patients.

Disclosure: Nothing to declare

\section{NO013.}

\section{Transition Strategy: From Pediatric to Adult Health Care and Long Term Follow-up}

Julia Ruiz Pato ${ }^{1}$, Marta Ausín Fernández, ${ }^{1}$ Ana Aida Casado Pardo ${ }^{1}$, Rebeca García Heras ${ }^{1}$, Laura Lozano Ferreiro ${ }^{I}$, Pilar Herreros López ${ }^{I}$, Miguel Ángel Diaz Perez $^{1}$

\section{${ }^{1}$ Hospital Infantil Universitario Niño Jesús, Madrid, Spain}

Background: Transplant is a complex process; long term follow-up is an integral part of Hematopoietic stem cell 
transplantation (HSCT) care which ensures surveillance and intervention for early and late complications. The number of long-term survivors has increased. There is a wide range of late effects, an increased burden of serious chronic conditions and impairments, involving organ systems and overall quality of life. Standardized mortality ratio at 15 years after HSCT remains 2.2-fold that of the general population.

Patients are lost for follow-up, adolescents and young adults have the highest probability of follow-up termination, decreasing opportunities to prevent or limit progression of late effects.

Our center is a national referral center for pediatric transplant. Patients continue their annual follow-up in our center up to the age of 18 , if no acute problem is present, and afterwards are referred to an adult center or their referral center. Our aim is to keep patients engaged with the longterm follow-up consultation when transition to adult center occurs.

Methods: Patients discharged to adult center due to age of majority receiving a HSCT between 2007 and 2014, were telephone contacted to know if follow-up continued after transition, and about their health condition.

Results: A total of 40 patients were discharged to adult centers in these years. Data collected after telephone contact: 26 patients $(65 \%)$ continued annual follow-up. The median of patients referred to adult centers each year was 4,5 with (range 10 to 2). The number of transitions to adult centers increases as we go back in the year of transplantation.

2 patients $(5 \%)$ did not continue follow-up by their own decision, 4 patients (10\%) were lost for follow-up due to wrong telephone number, no possible contact, and 8 patients $(20 \%)$ were pending to contact back to achieve direct information, contact had been made with parents.

Most of the patients continued their follow-up in an adult center, although there is a clear $15 \%$ of lost for follow-up patients.

A new strategy has been developed by transplant team, long term follow-up (LTFU) nurse consultant will keep record of discharged patients.

LTFU nurse consultation was developed one year ago and attends patients with an annual follow-up, engaging patients and family in their care plans. This may decrease number of patients lost for follow-up due to wrong contacts and also avoid losing patients that consider that there is no need to continue follow-up.

The appropriate center will be contacted and transition agreed to assure that adult center is also committed with LTFU. An updated patient report, long term effects and follow-up recommendations will be forwarded. LTFU nurse will facilitate transition and contact patient and/or center, to assure that care plans and follow-up is taking place, keeping contact information updated.

Conclusions: Importance of LTFU requires educating patients, family, and pediatric and future adult health care providers.

Planned transition to adult or referral centers will lead to a better compliance with LTFU

Future studies are needed to know about patients' unmet needs when transition occurs, and to evaluate the effectiveness of this new strategy.

Disclosure: Nothing to declare

\section{NO014.}

\section{Caregiver Education and Training for Self Administration of Oral Drugs in a Paediatric Center}

\author{
Giuseppe Marco Deiana ${ }^{I}$, Evelina Olcese ${ }^{I}$, Simona \\ Calza $^{I}$, Paolo Petralia ${ }^{1}$, Silvia Scelsi ${ }^{1}$
}

${ }^{1}$ Children Hospital G. Gaslini, Genova, Italy

Background: Literature suggests that effective patient education can influence cost savings and improve patient outcomes (CCO, 2006).

Stenberg et al. (2016) found that patients who participated in educational programs had better symptom control and improved self-management

Pidala, Anasetti, and Jim (2009) specifically note the importance of the role of patient education in improved quality of life in the complex autologous stem cell transplant patient population. A partnership involving education needs to be developed between the health care team and patients during all treatment stages to help mitigate and manage the risk of side effects. Additionally, the family or identified support people should be included in the education (CCO, 2015). In the pediatric fields parents are included in education.

Nursing staff provide education to patients and families through the assessment of learning needs, incorporating the teach-back method to assess comprehension, and documenting the care provided.

The objectives of this study were to design a structured education programme on administration of oral drug at home for caregiver and to evaluate the effectiveness of the programme.

Methods: We started in 2011 trying to understand what the main educational needs of caregivers and their families were in different areas. We evaluated their satisfaction and the reduction of hospital returns after the educational program. 
In 2016 we conducted a retrospective dual case-control study to understand if education, limited to the administration of oral drugs at home, had been effective.

The Kirkpatrick's evaluation model was used for the evaluation of the program. Indicators of effectiveness were selected based on Kirkpatrick's Training Evaluation Model, including satisfaction with the program, knowledge, attitude, problems and incident at home in administration.

The caregivers evaluated the program in terms of constituents of the program, utilization, and contribution to learning.

Twenty caregivers on a voluntary basis responded to the evaluation forms after the educational program. Data are evaluated using both quantitative and qualitative tools. SPSS (version 21) used for quantitative data for determining mean and standard deviation values. Descriptive qualitative analysis approach is used for the analysis of open-ended questions.

Results: The process allows us to always keep in check what the caregivers know, and they have a greater availability and confidence in every moment of the therapeutic programme. Satisfaction with the programme was high.

The evaluation of their progress makes the parents safer and they go home more relaxed.

In the study of 2016, there was a reduction of about $30 \%$ (260 vs 370) in the outpatient clinic accesses of the 15 families educated with the new process, compared to the other 15 educated with the old empirical method.

Conclusions: Family caregivers are increasingly important in the effective treatment and care of patients with cancer or subjected to bone marrow transplantation, including the necessary adherence to drug intake at home.

Disclosure: Nothing to declare

\section{NO015.}

\section{Sexual Health for Young People undergoing Haematopoietic Stem Cell Transplantation}

\author{
Lisa Burnett ${ }^{1}$, Kayleigh Douglas ${ }^{1}$ \\ ${ }^{1}$ Newcastle Upon Tyne NHS Foundation Trust, Newcastle \\ Upon Tyne, United Kingdom
}

Background: Sexual health and education is a fundamental part of holistic care when nursing young patients. Due to their complex health needs while undergoing Haematopoietic Stem Cell Transplant, their sexual health and education differs from other groups of young people.

Methods: We found that adult immunology and paediatric oncology services provide sexual health information for their patients during their transplant and treatment process. We recognised that these resources were not suitable for the paediatric immunology patients within our unit due to the differing isolation guidelines. This motivated us to participate in sexual health training and from this we were able to develop a sexual health service within the unit. This enabled the young people to receive in house education around their sexual health needs. The young patients were asked through discussions, what sexual health information they required. We then approached other HSCT units in the UK, sexual health services and our own medical team to discuss our findings. This then allowed us to formulate a sexual health booklet for pre and post Haematopoietic Stem Cell Transplant patients and a staff training resource.

Results: During this process, the young people disclosed that accessing sexual health information was difficult prior to the division of the booklet as they felt unable to discuss these matters confidently with staff. The booklet has allowed young people to access the information privately and is empowering our young patients in looking after their own sexual health. Following on from the success of the booklet, we were able to introduce a $\mathrm{C}$ - Card outlet into the unit which provides reproductive and sexual health information and access to free condoms if they wish. The scheme also allows us to signpost young people to their local services once returning home following treatment. For our long term patients, a new transitional service has been set up. Our sexual health service has contributed to ensuring the very best care is provided to the young users in the transitional clinic and meeting holistic needs.

Conclusions: Our poster will provide further information on the developing service of sexual health education and information for young people on our unit. This will display the journey of how we implemented a sexual health service in our unit, provide details of the booklet and feedback from the current and long term patients.

Disclosure: None declared. 